Revista Brasileira de Agricultura Irrigada v.11, nº.7, p. 2063 - 2075, 2017

ISSN 1982-7679 (On-line)

Fortaleza, CE, INOVAGRI - http://www.inovagri.org.br

DOI: $10.7127 /$ rbai.v11n700683

Protocolo 683.17 - 16/05/2017 Aprovado em 14/09/2017

\title{
MODELAGEM DA DISTRIBUIÇÃO DE ÁGUA DE MICROASPERSORES
}

\author{
Daniela D’Orazio Bortoluzzi ${ }^{1}$, Giuliani do Prado ${ }^{2}$
}

\begin{abstract}
RESUMO
O trabalho, realizado na Universidade Estadual de Maringá, em Cidade Gaúcha/PR, objetivou determinar e modelar a distribuição de água do microaspersor Naan Hadar ${ }^{\circledR}, 7110$. As condições ensaiadas foram quatro diâmetros de bocais $(0,9 ; 1,0 ; 1,1$ e 1,2 mm), dois insertos (grande e médio) e três pressões de serviço (98; 147 e $196 \mathrm{kPa}$ ). A distribuição de água foi coletada através de malha de coletores e as intensidades de precipitação foram empregadas para determinação dos raios de alcance e dos perfis radiais. Cada perfil radial foi adimensionalizado, expressado a distância ao microaspersor em termos da fração do raio e a intensidade em termos da intensidade média. Para as diferentes combinações de pressões e bocais, nos insertos de grande e médio alcance, a faixa de raio variou, respectivamente, entre: 2,5 a 4,1 m e 2,8 a 4,8 m. Devido a influência da haste do microaspersor, para cada quadrante da malha de coletores, foram ajustadas equações polinomiais de raio de alcance em função da pressão de serviço e diâmetro de bocal, que apresentaram coeficientes de determinação entre 0,689 a 0,894. A racionalização dos perfis radiais, aplicando o algoritmo K-Means, conferiu para cada quadrante, a ocorrência de seis formas geométricas típicas. O modelo de simulação da distribuição de água apresentou coeficiente de determinação de 77,12\% entre os valores de intensidade observados e simulados. Ao simular a distribuição de água, condições operacionais de pressão de serviço de $147 \mathrm{kPa}$, associadas a extensões efetivamente irrigadas entre 80 e $90 \%$ do raio de alcance, promoveram melhores uniformidades.
\end{abstract}

Palavras-Chave: perfil radial, raio de alcance, intensidade de aplicação

\section{MODELING WATER DISTRIBUTION OF MICRO-SPRINKLERS}

\begin{abstract}
This work was carried out at State University of Maringá, in Cidade Gaúcha/PR and it aimed to set up and modeling the water distribution of a Naan Hadar ${ }^{\circledR}, 7110$ micro-sprinkler. The test conditions were four nozzle diameters $(0.9,1.0,1.1$ and $1.2 \mathrm{~mm})$, two insert types (medium and extra range) and three working pressures (98; 147 and $196 \mathrm{kPa}$ ). The water distribution was collected from a square grid of collectors and the application rates were

\footnotetext{
${ }^{1}$ Engenheira Agrícola, Aluna de Mestrado do Programa de Pós-graduação em Agronomia da Universidade Estadual de Maringá, Maringá-PR, CEP: 87.020-900, e-mail: dani_dorazio@hotmail.com.

${ }^{2}$ Engenheiro Agrônomo, Professor Associado A do Departamento de Engenharia Agrícola da Universidade Estadual de Maringá, Cidade Gaúcha-PR, CEP: 87.820-000, e-mail: gprado@uem.br.
} 
employed to set up the radius of throw and the water distribution curves. Each water distribution curve was normalized, expressing the distance from micro-sprinkler and the application rate, respectively, by the fraction of radius of throw and average application rate. For the different combinations of pressures and nozzles, extra and medium range inserts presented, respectively, radius of throw from: 2.5 to $4.1 \mathrm{~m}$ and 2.8 to $4.8 \mathrm{~m}$. Due to the influence of micro-sprinkler's bridge, for each collector grid quadrant, were adjusted polynomial equation of radius of throw as function of working pressure and nozzle diameter, which presented determination coefficient from 0.689 to 0.894. Applying the K-Means algorithm to the normalized radial legs was identified six curves of different geometric shapes for each quadrant. The water distribution simulation model to micro-sprinklers presented a determination coefficient of $77.12 \%$ between application rate observed and simulated. By simulating water distribution, pressure working conditions of $147 \mathrm{kPa}$, associated with effectively irrigated area around 80 and $90 \%$ of the radius of throw, gave the best water uniformity values.

Keywords: distribution curve, radius of throw, application rate

\section{INTRODUÇÃO}

A produção agrícola irrigada possui considerável demanda de água devido às exigências hídricas de cada cultura e por serem produzidas em grandes extensões de terras (PRADO; COLOMBO, 2013). Quando bem planejada, torna-se um instrumento de elevação de renda, oportunidade de emprego, economia de recursos, além de expandir a oferta de produtos agrícolas (FRIZZONE et al., 2012).

Dentre os sistemas pressurizados, a microirrigação, que enquadra a irrigação por gotejamento e por microaspersão, é a que apresenta maior eficiência, uma vez que as perdas na aplicação de água são menores (MACEDO et al., 2010). A microaspersão é um sistema de irrigação pela qual a água é aplicada de forma pulverizada por meio de microaspersores que operam com pressão menor que $207 \mathrm{kPa}$, vazão de 20 a $100 \mathrm{~L} \mathrm{~h}^{-1} \mathrm{e}$ sua área molhada apresenta forma de discos ou faixas molhadas abaixo das copas das plantas, com diâmetro de alcance dos emissores variando de 1,5 a 10 m (BERNARDO et al., 2006).

No mercado há uma grande variedade de microaspersores que permitem uma grande amplitude de vazões (diâmetro dos bocais), alcances (tipos de insertos) e formas de montagem, que atendem tanto campo aberto quanto estufas. Em ambientes protegidos, os microaspersores são normalmente utilizados em conjunto, invertidos e suspensos para facilitar o manejo. Entretanto, para condições de campo, normalmente os microaspersores operam isoladamente sem sobreposição lateral. Assim, o perfil de distribuição de água e a quantidade de água aplicada, são parâmetros essenciais para o correto manejo e dimensionamento do sistema de irrigação (NASCIMENTO et al., 1999; SANDRI et al., 2010).

As características de distribuição de água dos microaspersores dependem do modelo do microaspersor e de condições operacionais como: diâmetro dos bocais; inclinação do jato; pressão de serviço; altura de instalação e; estabilidade da haste de sustentação do emissor (ANDRADE, 2013). Por efeito desses fatores, a realização de ensaios a campo torna-se uma tarefa trabalhosa e demorada, e geralmente, não é possível a realização de todas as combinações desejadas.

Os perfis de distribuição de água de emissores são de suma importância para pesquisa, controle de qualidade e avaliação do equipamento. Entretanto, a maioria dos fabricantes não disponibilizam nos catálogos técnicos algumas características importantes como o perfil de distribuição de água para as diferentes combinações de bocais, pressão de serviço (PRADO; COLOMBO, 2005) e tipo de inserto. Esses dados poderiam ser 
empregados em simulações digitais para determinar as melhores condições operacionais do microaspersor.

Conforme Solomon e Bezdek (1980), a adimensionalização de perfis radiais consiste em expressar a distância ao emissor em termos do raio de alcance e a respectiva intensidade de precipitação, em termos da intensidade média. Esse procedimento permite identificar perfis adimensionais que apresentem formas geométricas semelhantes empregando o algoritmo de agrupamento KMeans. Assim, é possível avaliar e simular a distribuição de água de microaspersores operando com diferentes combinações de pressão, diâmetro de bocais e insertos através da racionalização dos perfis radiais, a fim de reduzir o número de ensaios.

Deste modo, considerando a falta de informação de algumas características operacionais importantes do microaspersor Naan Hadar ${ }^{\circledR}$, modelo 7110 , este trabalho teve por objetivo levantar e modelar a distribuição de água do microaspersor empregando o algoritmo de agrupamento KMeans.

\section{MATERIAL E MÉTODOS}

O trabalho foi realizado na Universidade Estadual de Maringá - UEM, em Cidade Gaúcha, PR. Nesse estudo foram avaliadas e modeladas as características de distribuição de água, raio de alcance e vazão do microaspersor Naan Hadar ${ }^{\circledR}$, modelo 7110 .

Para o desenvolvimento dos ensaios foi montada uma bancada nas proximidades do Laboratório de Hidráulica e Irrigação da UEM. Essa banca foi constituída de: a) fonte de captação de água; b) conjunto motobomba; c) tubulação dotada de registro de gaveta, filtro e hidrômetro; d) manômetro analógico, acoplado na tomada de pressão instalado no tubo de subida do microaspersor e; e) malha de coletores de água.

A vazão, o raio de alcance e a distribuição de água do microaspersor foram determinados para 24 diferentes condições operacionais distintas, dadas pelas combinações de: i) três pressões de serviço (98, 147 e $196 \mathrm{kPa})$; ii) quatro bocais com diferentes diâmetros (0,9 mm - cinza; $1,0 \mathrm{~mm}$ - roxo; $1,1 \mathrm{~mm}$ - vermelho e; $1,2 \mathrm{~mm}$ alaranjado) e; iii) dois tipos de insertos (médio e longo alcance).

Os ensaios de distribuição de água do microaspersor foram realizados empregando o método da malha de coletores de água (Figura 1a), conforme norma técnica ISO 8026 (ISO, 1995), onde os coletores foram distribuídos em torno do microaspersor com um espaçamento de $0,9 \times 0,9 \mathrm{~m}$. Para as avaliações, instalou-se o microaspersor mantendo-se uma distância vertical de 0,34 m entre o bocal e o plano da área de captação dos coletores. Esses coletores plásticos, com diâmetro de captação igual a $0,08 \mathrm{~m}$, foram instalados em área plana coberta e os ensaios foram realizados na condição de ausência de ventos.

Para aferição da pressão de operação do microaspersor, com auxílio de registro de gaveta, utilizou-se um manômetro instalado a altura do bocal do microaspersor. Durante os ensaios, as pressões no manômetro foram verificadas a cada 15 minutos e a vazão aplicada foi mensurada com auxílio de hidrômetro. Cada ensaio teve duração de uma hora e os volumes de água contidos nos coletores foram transformados em intensidade de aplicação de água.

Conforme sugestões de Gat e Molle (2000), ao término de cada ensaio, o valor de vazão determinado pelo hidrômetro foi comparado com o valor de vazão coletada. A vazão coletada corresponde ao somatório do produto das intensidades de precipitação, observadas em cada coletor, multiplicada pela área de influência de cada coletor $(0,9$ x 0,9 = $0,81 \mathrm{~m}^{2}$ ), dada por:

$$
\mathrm{Q}_{\mathrm{c}}=\Delta \mathrm{e}^{2} \cdot \sum_{\mathrm{j}=1}^{\mathrm{n}} \sum_{\mathrm{k}=1}^{\mathrm{n}} \mathrm{i}_{\mathrm{j}, \mathrm{k}}
$$

em que: Qc é a vazão coletada na malha de coletores $\left(\mathrm{L} \mathrm{h}^{-1}\right) ; \mathrm{i}_{\mathrm{j}, \mathrm{k}} \quad \mathrm{a}$ intensidade de precipitação observada em um coletor de 


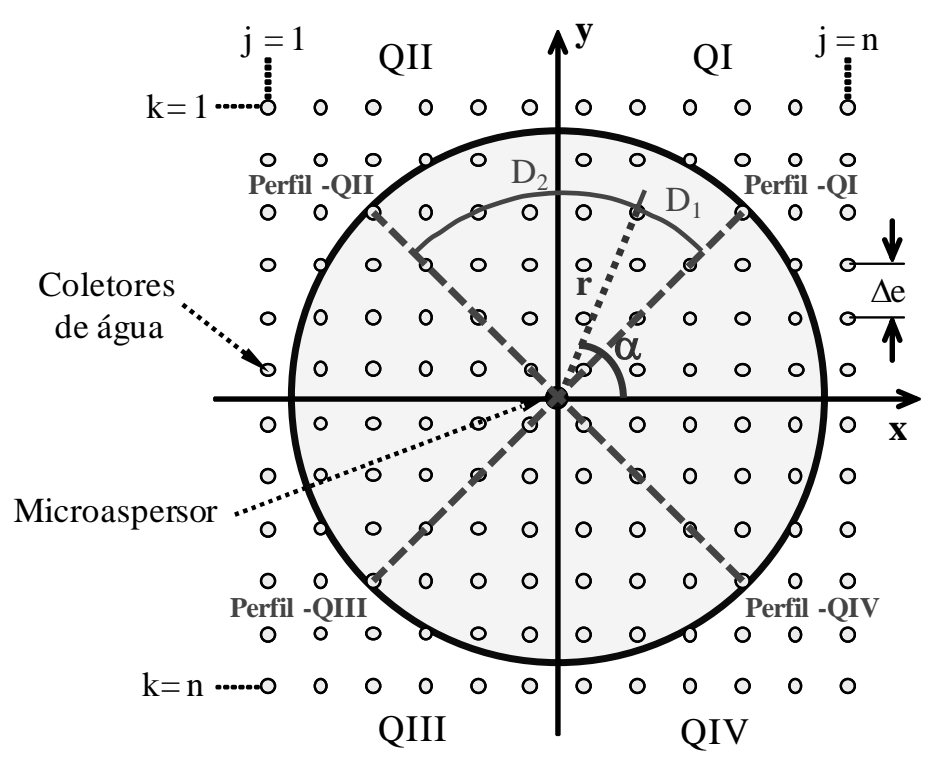

(a)

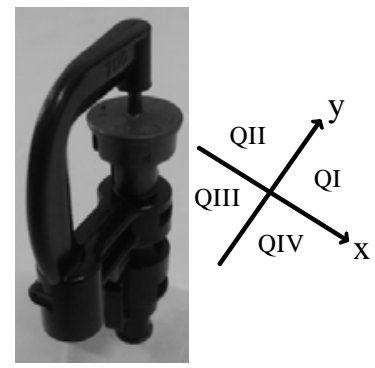

Posiçã o do microa spersor na malha de coletores

(b)

Figura 1. Disposição dos coletores de água (a) e posicionamento do microaspersor (b) no sistema cartesiano de coordenadas (x, y).

Os dados de intensidade de precipitação corrigidos, pela relação entre a vazão aplicada pela coletada, foram usados para construir os perfis radiais do microaspersor por quadrante (QI, QII, QIII e QIV) da malha de coletores (Figura 1a), orientada conforme a posição do

$$
\mathrm{ip}_{\mathrm{s}}=\frac{\sum_{\mathrm{j}=1}^{\mathrm{n}} \sum_{\mathrm{k}=1}^{\mathrm{n}} \mathrm{ic}_{\mathrm{j}, \mathrm{k}}(\mathrm{r})}{\mathrm{m}}
$$

em que: ips é a intensidade de precipitação no perfil radial de um quadrante, a uma distância radial de r e num ponto de amostragem s (mm $\left.\mathrm{h}^{-1}\right) ; i c_{\mathrm{j}, \mathrm{k}}(\mathrm{r})$ a intensidade de precipitação em ponto de amostragem de mesma distância radial $\mathrm{r}\left(\mathrm{mm} \mathrm{h}^{-1}\right)$; $\mathrm{r}$ a distância radial do coletor ao microaspersor (m); m o número de pontos de amostragem localizados a mesma distância radial $\mathrm{r}$ e; $\mathrm{x}$ e $\mathrm{y}$ coordenadas cartesianas da malha de coletores (m).

Os perfis de distribuição de água, representativos de cada quadrante (Figura 1a), foram adimensionalizados de acordo com a metodologia apresentada por Solomon e Besdek (1980), em que as distâncias ao microaspersor foram expressas em termos de fração do raio de alcance (Equação 3), e os microaspersor (Figura 1b). Para esse procedimento, foi determinada a média dos valores de intensidades de precipitação observados a mesma distância radial ao microaspersor em cada quadrante da malha de coletores, dado por:

valores de intensidade de aplicação expressas em termos da fração da intensidade média de aplicação de água (Equação 4). $\mathrm{Na}$ adimensionalização foi empregado o raio de alcance, ponto mais distante onde ocorre a intensidade de $0,13 \mathrm{~mm} \mathrm{~h}^{-1}$ ISO 8026 (ISO, 1995), e a intensidade média, ambos obtidos por quadrante.

$$
\begin{gathered}
\mathrm{ra}_{\mathrm{s}}=\frac{\mathrm{r}_{\mathrm{l}}}{\mathrm{R}_{\mathrm{Q}}} \\
\mathrm{ia}_{\mathrm{s}}=\frac{\mathrm{ip}_{\mathrm{l}}(\mathrm{r}) \cdot \pi \cdot \mathrm{R}_{\mathrm{Q}}{ }^{2}}{1000 \cdot \mathrm{Q}_{\mathrm{c}}}=\frac{\mathrm{ip}_{\mathrm{s}}(\mathrm{r})}{\mathrm{im}_{\mathrm{Q}}}
\end{gathered}
$$


em que: ras é a fração do raio de alcance do microaspersor (adimensional); ias a fração da intensidade média de aplicação de água (adimensional); $\mathrm{R}_{\mathrm{Q}}$ o raio de alcance do microaspersor (m); imo a intensidade média de aplicação de água $\left(\mathrm{mm} \mathrm{h}^{-1}\right)$.
Aos valores de raio de alcance, para cada quadrante e tipo de inserto, e vazão aplicada, em função do diâmetro do bocal e pressão de serviço, foram ajustados os coeficientes das equações de regressão:

$$
\begin{gathered}
R_{\text {est }}=a_{1} \cdot p^{2}+a_{2} \cdot p+a_{3} \cdot p \cdot b+a_{4} \cdot b^{2}+a_{5} \cdot b+a_{6} \\
Q_{\text {est }}=a_{7} \cdot b^{a_{8}} \cdot p^{a_{9}}
\end{gathered}
$$

em que:

Rest é o raio de alcance estimado (m); p a pressão de serviço do microaspersor (kPa); b o diâmetro do bocal (mm) e; a1, a2, $a_{3}, a_{4}, a_{5}, a_{6}, a_{7}, a_{8}$ e a9, são constantes de ajuste.
Com os dados obtidos em cada perfil radial foram gerados através do interpolador spline cúbico (BURDEN; FAIRES, 2003) vinte valores de intensidade adimensional de precipitação correspondente as vinte frações do raio de alcance (rat) dadas por:

$$
\mathrm{ra}_{\mathrm{t}}=0,025+(\mathrm{t}-1) \cdot 0,05, \text { com } 20 \geq \mathrm{t} \geq 1
$$

A fim de identificar formas geométricas típicas assumidas pelos perfis radiais adimensionais, os vinte e quatro perfis de cada quadrante foram submetidos à análise de agrupamento utilizando o algoritmo K-Means (PRADO; COLOMBO, 2005).
Para simular a distribuição de água aplicada pelo microaspersor, foi considerado que a influência das intensidades de aplicação de água dos perfis radiais representativos de cada quadrante (Figura 1a), definida pelo ângulo $\alpha$, são dadas pelos perfis:

$$
\text { Perfis }=\left\{\begin{array}{l}
\text { se : } \frac{3 \pi}{4}>\alpha \geq \frac{\pi}{4} \rightarrow \text { Perfil - QI e Perfil - QII } \\
\text { se: } \frac{5 \pi}{4}>\alpha \geq \frac{3 \pi}{4} \rightarrow \text { Perfil - QII e Perfil - QIII } \\
\text { se: } \frac{7 \pi}{4}>\alpha \geq \frac{5 \pi}{4} \rightarrow \text { Perfil - QIII e Perfil - QIV } \\
\text { se : } 2 \pi>\alpha \geq \frac{7 \pi}{4} \text { ou } \frac{\pi}{4}>\alpha \geq 0 \rightarrow \text { Perfil - QIV e Perfil - QI }
\end{array}\right.
$$

em que: Perfis são os perfis radiais que definem a intensidade de aplicação de água em cada quadrante e; $\alpha$ o ângulo de abertura entre o coletor e a origem do eixo cartesiano (radiano).

Ao dimensionalizar os perfis radiais de cada quadrante e durante a aplicação de água, cada coletor está alocado a distância radial " $\mathrm{r}$ " ao microaspersor e a uma distância angular $D_{1}$ e $D_{2}$, em relação aos perfis representativos de cada quadrante (Figura 1a). Em função do ângulo $\alpha$, essas distâncias angulares são dadas por: 


$$
\mathrm{D}_{1}=\left\{\begin{array}{l}
\text { se } \frac{3 \pi}{4}>\alpha \geq \frac{\pi}{4} \rightarrow \mathrm{D}_{1}=\mathrm{r} \cdot\left(\alpha-\frac{\pi}{4}\right) \\
\text { se }: \frac{5 \pi}{4}>\alpha \geq \frac{3 \pi}{4} \rightarrow \mathrm{D}_{1}=\mathrm{r} \cdot\left(\alpha-\frac{3 \pi}{4}\right) \\
\text { se: } 2 \pi>\alpha \geq \frac{7 \pi}{4} \rightarrow \mathrm{D}_{1}=\mathrm{r} \cdot\left(\alpha-\frac{5 \pi}{4}\right) \\
\text { se: } \frac{\pi}{4}>\alpha \geq 0 \rightarrow \mathrm{D}_{1}=\mathrm{r} \cdot\left(\alpha+\frac{\pi}{4}\right) \\
\mathrm{D}_{2}=\mathrm{r} \cdot \frac{\pi}{2}-\mathrm{D}_{1}
\end{array}\right.
$$

em que: $\mathrm{D}_{1}$ é a distância angular do coletor ao perfil radial anterior $(\mathrm{m}) ; \mathrm{D}_{2}$ a distância angular do coletor ao perfil radial posterior (m) e; r a distância radial do coletor ao microaspersor (m).

A intensidade de precipitação média aplicada em cada coletor foi determinada ponderando-se a intensidade de precipitação, obtida em cada perfil radial de cada quadrante a uma distância "r" (Figura 1a), pelo inverso do quadrado da distância angular ao coletor (MELLO et al., 2003).

De modo a verificar a adequação entre as intensidades observadas e simuladas pelo modelo, que apresentavam diferenças absolutas maiores que zero, na malha de coletores, foi realizada as seguintes análises: i) determinação da média do desvio absoluto e do valor do coeficiente de determinação $\left(\mathrm{R}^{2}\right)$ da reta ajustada aos pares (simulados e observados) e; ii) análise do índice de confiança de desempenho (c) de Camargo e Sentelhas (1997) que, conforme Conceição e Coelho (2003), é dado pela multiplicação do coeficiente de correlação (r) pelo índice de Willmott (d).

A partir do modelo de simulação da distribuição de água, foi estabelecida uma malha de coletores de água de $0,20 \times 0,20 \mathrm{~m}$ e simulada as intensidades de aplicação de água em torno do microaspersor para as 24 condições operacionais de diâmetro de bocal, pressão de serviço e tipo de inserto. Os dados de intensidade de aplicação de água simulados foram empregados para o cálculo da uniformidade de aplicação de água de Christiansen (CUC), considerando raios de alcance (R) efetivamente irrigados médios de: $1 R, 0,9 R, 0,8 R$ e $0,7 R$.

\section{RESULTADOS E DISCUSSÃO}

Na Figura 2 são apresentados os valores de vazão versus pressão de serviço dos quatro bocais do microaspersor ensaiado. Ao ajustar a equação potencial de vazão, em função da pressão de serviço e do diâmetro do bocal, o microaspersor apresentou expoente, para a variável independente pressão, próximo a 0,5, classificando esse microaspersor como um emissor de regime turbulento (CABELLO, 1996; FRIZZONE et al., 2012). Dantas Neto et al. (1997), ao ensaiar o microaspersor NAAN 7110 com o bocal laranja, também encontraram expoentes de vazão próximo a 0,5. Na equação ajustada (Figura 2), o alto coeficiente de determinação $\left(\mathrm{R}^{2}=99,48 \%\right)$ indica a adequação dos dados ao modelo potencial. 


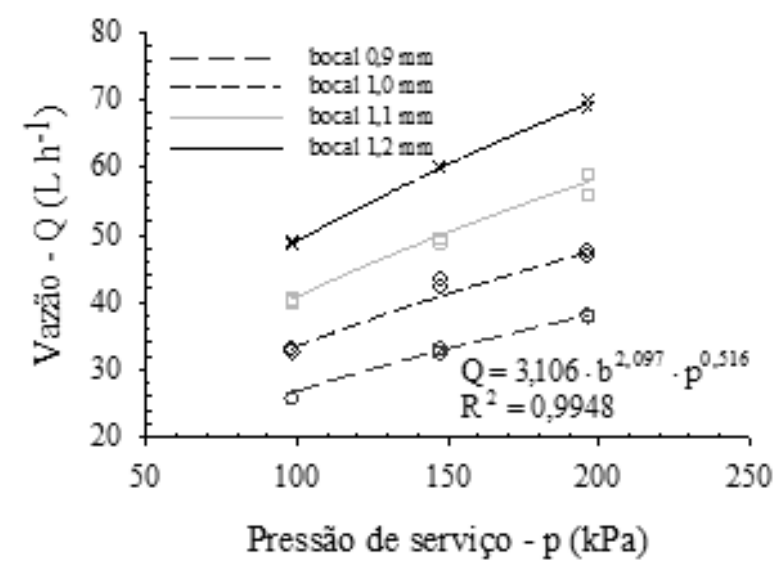

Figura 2. Vazão do microaspersor em função da pressão de serviço e para diferentes bocais.
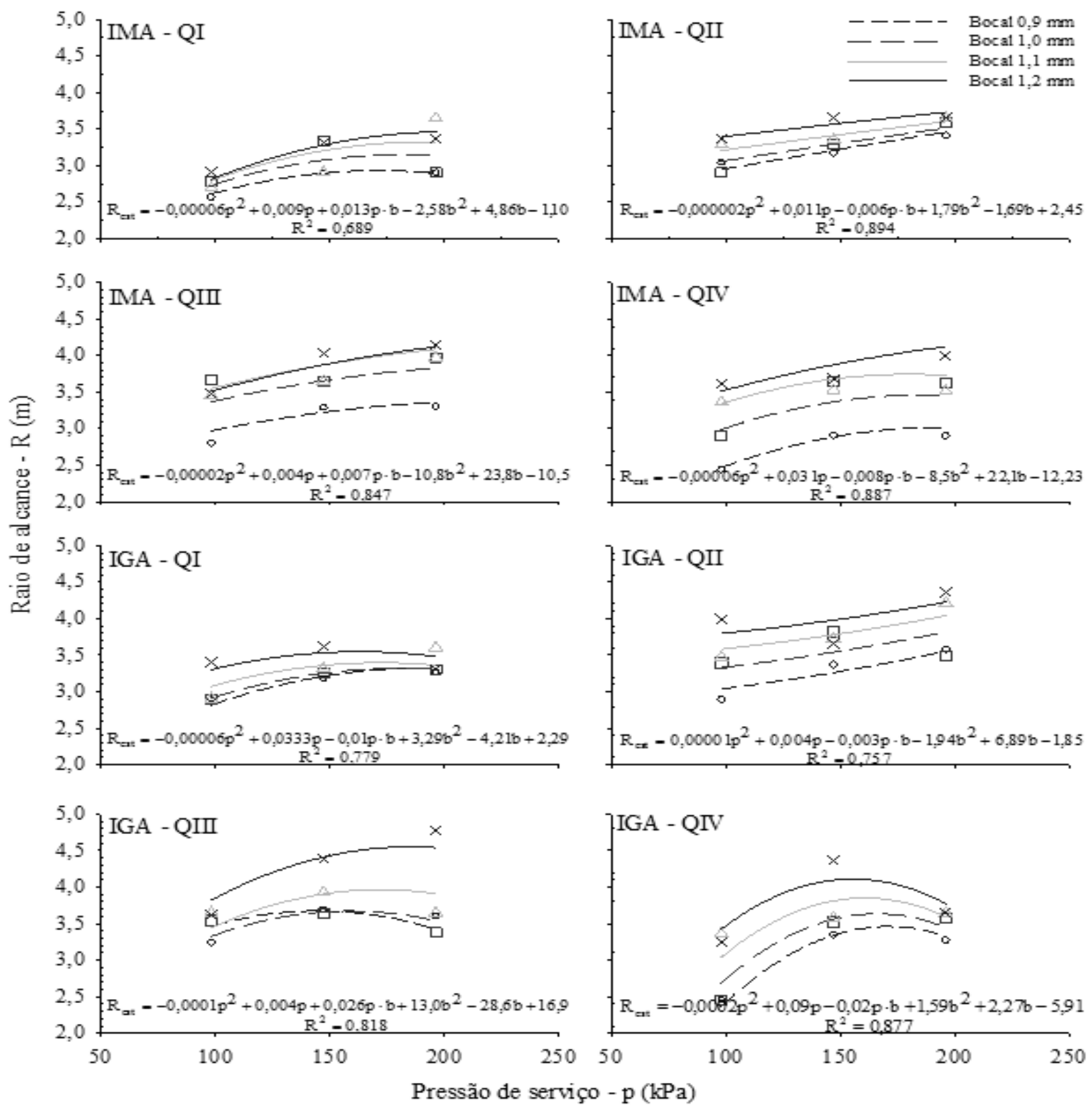

Figura 3. Raio alcance em função da pressão de serviço, diâmetro de bocal e inserto (médio - IMA e grande - IGA alcance) nos diferentes quadrantes (Q) da malha de coletores. 
Os raios de alcance do microaspersor variaram entre 2,5 a 4,1 m e 2,8 a 4,8 m, respectivamente, para os insertos de médio e grande alcance, e houve um aumento no raio de alcance com incrementos no diâmetro do bocal (Figura 3). O incremento no raio de alcance, devido ao diâmetro de bocal, também ficou evidenciado nos perfis de distribuição de água de microaspersores ensaiados por Santos et al. (2009).

Os insertos de grande alcance proporcionam maiores raios de alcance que os insertos de médio alcance (Figura 3), gerando menores intensidades de aplicação de água. De modo geral, incrementos na pressão geraram um aumento no raio de alcance até um dado valor, a partir desse ponto, devido a pulverização do jato, há uma redução no raio (Figura 3). Esse comportamento foi retratado por Prado et al. (2008), em ensaios de aspersores e, como apresentado na Figura 3, equações polinomiais, em função do diâmetro do bocal e da pressão, apresentaram ajustes com coeficientes de determinação $\left(\mathrm{R}^{2}\right)$ superiores a $69 \%$.

Para os quatro quadrantes da malha de coletores, a variação do erro médio $\left(E_{k}\right)$, em função do número $\mathrm{K}$ de perfis típicos, ao aplicar o algoritmo de agrupamento K-Means está representada na Figura 4. Conforme

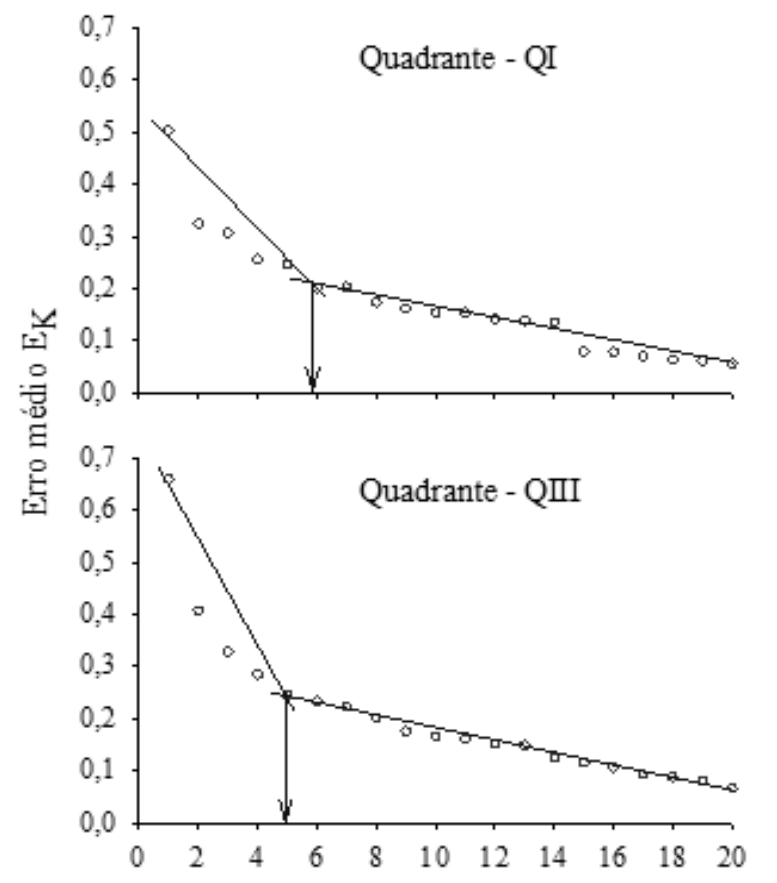

Solomon e Bezdek (1980), o número de perfis é escolhido através do ponto de inflexão entre o erro médio e o número $\mathrm{K}$ de perfis. Dessa forma, ao definir seis perfis radiais $(K=6)$ para cada quadrante, o erro médio na intensidade adimensional de precipitação variou entre 0,17 a 0,24 e, a partir desse ponto, com o aumento no número de "K" perfis, o erro médio tem um decréscimo constante até o valor de zero $(\mathrm{K}=24)$. Prado e Colombo (2005), agrupando 45 perfis radiais adimensionais de um canhão hidráulico em 3 perfis típicos, obtiveram um erro médio na intensidade adimensional de precipitação de 0,147. Entretanto, Prado (2016), ao agrupar 16 perfis radiais adimensionais de um aspersor de tamanho médio em 3 perfis típicos, obteve um erro médio igual a 0,19 .

Os 24 perfis adimensionais obtidos nos ensaios, para cada quadrante, sob condições de operações distintas, foram racionalizados gerando seis perfis típicos. Na Tabela 1 estão definidas as condições operacionais (pressão $\mathrm{x}$ bocal $\mathrm{x}$ inserto $\mathrm{x}$ quadrante) do microaspersor pertencentes a cada grupo (I, II, III, IV, V ou VI) e na Figura 5 são apresentadas as formas geométricas típicas assumidas por esses perfis radiais.
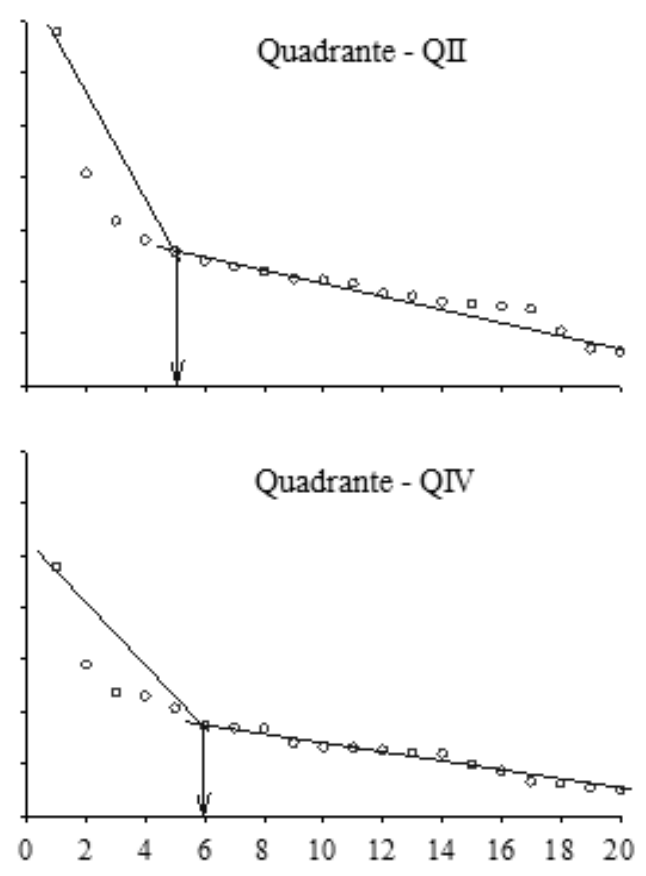

Número de grupos ou perfis - K

Figura 4. Erro médio $\left(E_{k}\right)$ na estimativa $\left.d_{j}\right)$ da aplicação de água em função do número $(K)$ de perfis típicos adotados. 
O decréscimo na intensidade de precipitação ao longo do perfil radial, perfis típicos A e B de Christiansen (1942), característica observada em microsapersores ensaiados por Andrade (2013), Dantas Neto et al. (1997) e Sandri et al. (2010), independente do quadrante, foi observado, principalmente, para o perfil típico VI (Figura 5). Conforme a Tabela 1, o perfil típico VI está associado a condições operacionais de pressão mais elevadas $(\mathrm{p}=196 \mathrm{kPa})$.

Condições operacionais que levam ao perfil radial típico E de Christiansen (1942), que é gerado por um acúmulo de água nos pontos finais do perfil radial (Figura 5), foram mais nítidas nos perfis típicos I, II e IV (Tabela 1). Esses perfis típicos tiveram maior ocorrência na pressão de serviço de $98 \mathrm{kPa}$, entretanto, o acúmulo de água em pontos próximo ao raio de alcance ficou mais evidente nos quadrante II e III. Segundo Prado et al. (2008), condições operacionais que geram perfil radial típico $\mathrm{E}$ de Christiansen (1942) proporcionam baixos valores de uniformidade de aplicação de água e devem ser evitadas.

Tabela 1. Perfis adimensionais típicos assumidos em função do número de grupos ou perfis (K) adotados, diâmetro de bocal $(0,9 ; 1,0 ; 1,1$ e; 1,2 mm), insertos de médio (IMA) e grande (IGA) alcance e quadrantes (QI; QII; QIII e; QIV).

\begin{tabular}{|c|c|c|c|c|c|c|c|c|c|}
\hline \multirow[t]{2}{*}{$\mathbf{Q}$} & \multirow{2}{*}{$\underset{(\mathbf{k P a})}{\mathbf{p}}$} & \multicolumn{2}{|c|}{$\begin{array}{c}\text { Bocal cinza } \\
(0,9 \mathrm{~mm})\end{array}$} & \multicolumn{2}{|c|}{$\begin{array}{c}\text { Bocal roxo } \\
(1,0 \mathrm{~mm})\end{array}$} & \multicolumn{2}{|c|}{$\begin{array}{c}\text { Bocal vermelho } \\
(1,1 \mathrm{~mm})\end{array}$} & \multicolumn{2}{|c|}{$\begin{array}{c}\text { Bocal alaranjado } \\
(1,2 \mathrm{~mm})\end{array}$} \\
\hline & & IMA & IGA & IMA & IGA & IMA & IGA & IMA & IGA \\
\hline & 98 & IV & I & $\mathrm{V}$ & IV & IV & I & IV & II \\
\hline \multirow[t]{2}{*}{$\mathbf{Q I}$} & 147 & V & IV & III & III & III & III & III & V \\
\hline & 196 & VI & III & III & II & VI & VI & III & III \\
\hline & 98 & IV & I & IV & I & IV & I & II & I \\
\hline \multirow[t]{2}{*}{ QII } & 147 & V & II & V & II & V & V & V & VI \\
\hline & 196 & VI & III & VI & V & VI & V & VI & VI \\
\hline \multirow{3}{*}{ QIII } & 98 & IV & I & II & I & I & I & II & I \\
\hline & 147 & $\mathrm{~V}$ & II & VI & II & V & II & V & V \\
\hline & 196 & VI & III & VI & VI & VI & V & VI & III \\
\hline \multirow{3}{*}{ QIV } & 98 & IV & I & III & IV & III & IV & III & IV \\
\hline & 147 & V & II & VI & V & V & II & V & V \\
\hline & 196 & VI & II & VI & VI & VI & VI & VI & VI \\
\hline
\end{tabular}

Os perfis típicos III e $\mathrm{V}$ apresentam maior uniformidade na intensidade de aplicação de água ao longo do perfil radial, principalmente, nos quadrante I, II e III assemelhando-se aos perfis $\mathrm{C}$ e $\mathrm{D}$ de Christiansen (1942). A ocorrência dessas duas formas geométricas típicas está associada, principalmente, a pressão de operação de 147
$\mathrm{kPa}$ (Tabela 1), pressão usualmente recomendada para microaspersão (FRIZZONE et al., 2012). Também, microaspersores operando com insertos giratório, normalmente, operam de forma individual e perfis mais uniformes melhoram a uniformidade de aplicação de água. 

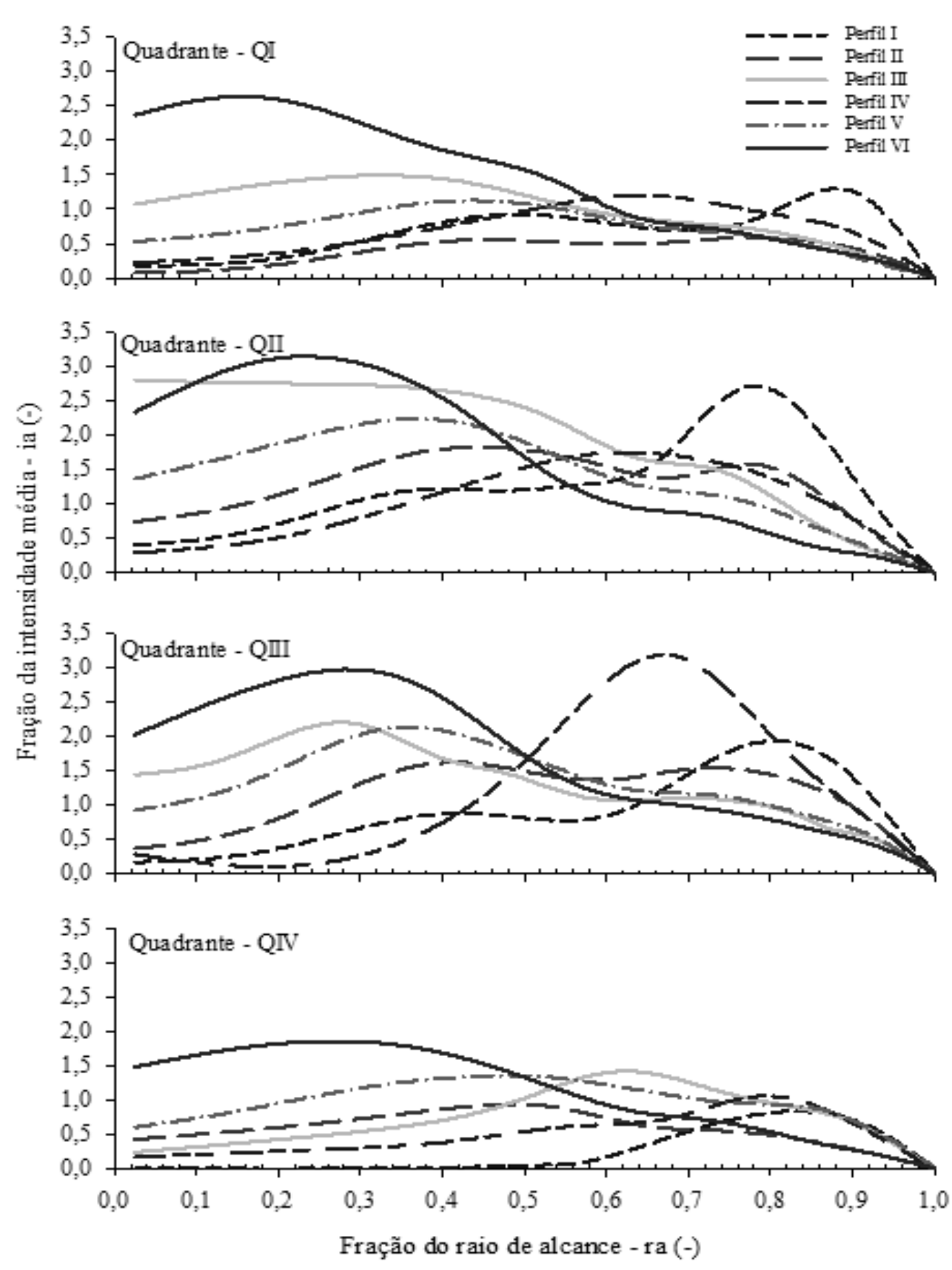

Figura 5. Formas geométricas típicas assumidas após o agrupamento dos perfis radiais adimensionais para cada quadrante da malha de coletores.

Na Figura 6 são apresentados os valores de intensidade de aplicação de água observados e simulados na malha de coletores para as diferentes condições operacionais. O erro absoluto médio na estimativa da intensidade de precipitação foi de $0,311 \mathrm{~mm}$ $\mathrm{h}^{-1}$, o coeficiente de determinação da reta ajustada de $77,2 \%$ e o índice de confiança de desempenho (c) de 0,817. Também, a reta ajustada com coeficiente angular próximo a unidade e coeficiente linear próximo a zero, revelou proximidade entre a reta $1: 1$.

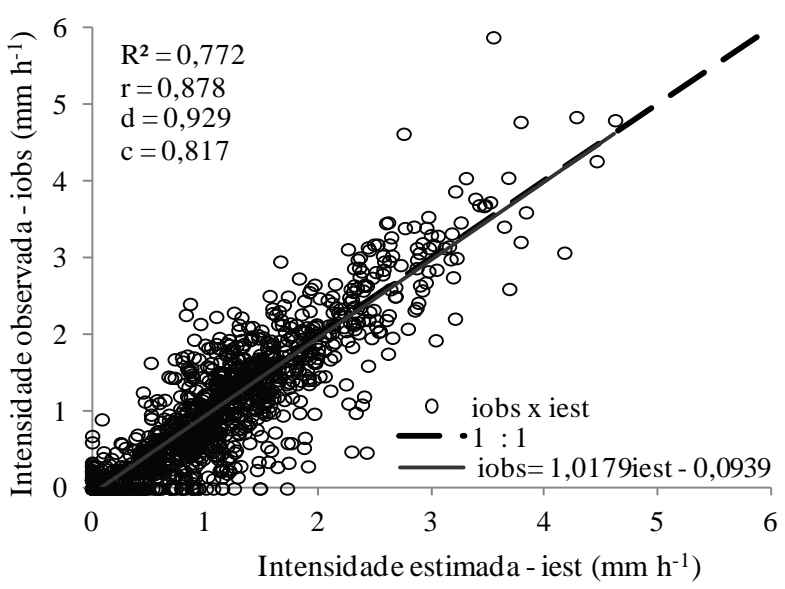


Figura 6. Valores de intensidade de precipitação obtidos por simulação e observados nos ensaios na malha de coletores de água.

O coeficiente de determinação encontrado $\left(R^{2}=0,772\right)$ está entre os valores de coeficientes de determinação obtidos por Montero et al. (2001), para a validação de um programa de simulação da distribuição espacial de água de aspersores. Também, o índice de confiança de desempenho de Camargo e Sentelhas (1997) obtido $(0,75 \leq \mathrm{c}$ $\leq 0,85$ ) é classificado como muito bom.

Os valores de uniformidade de
Christiansen (CUC), simulados para as diferentes condições operacionais e quatro diferentes raios efetivamente irrigados (1R, 0,9R, 0,8R e 0,7R) estão apresentados na Figura 7. Para microaspersores operando isoladamente, os valores de uniformidade determinados considerando toda a extensão do raio de alcance, como determinado nos trabalhos de Nascimento et al. (1999) e Sandri et al. (2010), geram baixos valores de CUC. Essa redução no valor de CUC (Figura 7) é mais acentuada para condições operacionais que levam ao perfil típico VI (Figura

5).

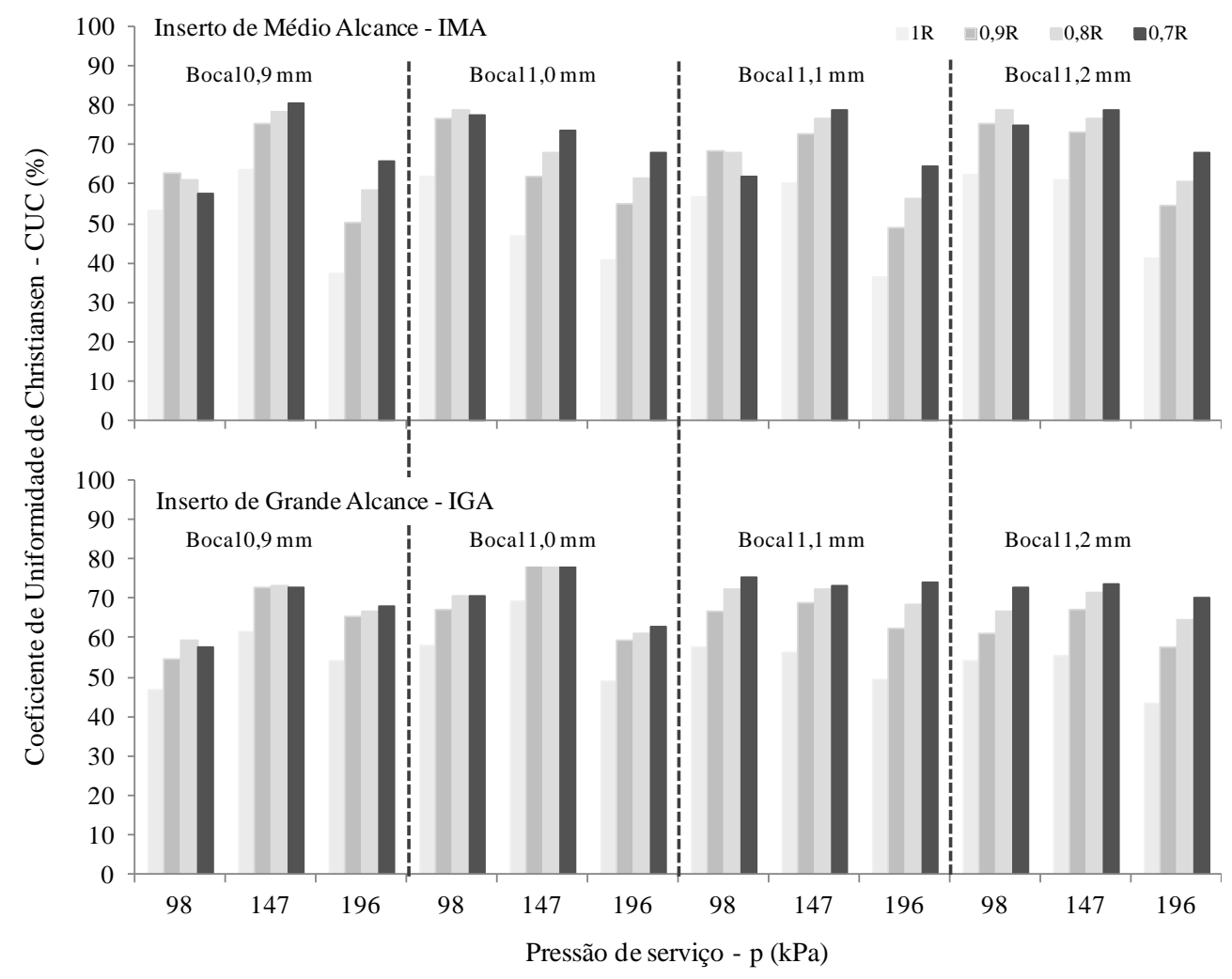

Figura 7. Uniformidade de aplicação de água de Christiansen em função de pressão de serviço, inserto, diâmetro do bocal e fração do raio de alcance.

Ao considerar raio efetivamente irrigado entre $0,8 \mathrm{R}$ e $0,9 \mathrm{R}$, a uniformidade apresentou valores mais altos, alcançando valores de CUC próximos a 80\%, principalmente, para condições operacionais de pressão iguais a $147 \mathrm{kPa}$ (Figura 7), que geram perfis radiais com forma geométrica mais uniforme (Figura 5). Keller e Bliesner (1990), para canhões hidráulicos em extremidade final de pivô central, sugerem um raio efetivamente irrigado igual a $0,75 R$, todavia, valores entre $0,7 \mathrm{R}$ e $0,8 \mathrm{R}$ não promoveram incrementos significativos nos valores de CUC (Figura 7).

\section{CONCLUSÕES}

Com base nos resultados obtidos conclui-se que: i) a haste influencia na distribuição de água do microaspersor; ii) perfis radiais com seis formas geométricas, 
para cada quadrante, podem ser empregados para representar as diferentes condições operacionais do microaspersor; iii) condições de trabalho do microaspersor operando na pressão de $147 \mathrm{kPa}$ promovem melhores uniformidades e; iv) raios efetivamente irrigados entre 0,8 a $0,9 \mathrm{R}$ geram altos índices de uniformidade para microaspersores operando isoladamente.

\section{REFERÊNCIAS}

ANDRADE, S. M. Desempenho hidráulico de microaspersor autocompensante, novo e usado. 2013. Dissertação (Mestrado em Agronomia/Ciência do Solo) - Universidade Estadual Paulista, Jaboticabal, 2013.

BERNARDO, S.; SOARES, A. A.; MANTOVANI, E. C. Manual de irrigação. 8.ed. Viçosa: UFV, 2006. 625 p.

BURDEN, R. L.; FAIRES, J. D. Análise numérica. São Paulo: Pioneira Thomson Learning, 2003. 740 p.

CABELLO, F. P. Riegos localizados de alta frecuencia (RLAF): goteo, microaspersión, exudación. 3.ed. Madrid: Mundi-Prensa, 1996. $513 \mathrm{p}$.

CAMARGO, A. P.; SENTELHAS, P. C. Avaliação do desempenho de diferentes métodos de estimativa da evapotranspiração potencial no Estado de São Paulo, Brasil. Revista Brasileira de Agrometeorologia, Santa Maria, v. 5, n. 1, p. 89-97, 1997.

CHRISTIANSEN, J. E. Irrigation by sprinkling. Berkeley: California Agricultural Station, 1942. 124 p. (Bulletin, 670).

CONCEIÇÃO, M. A. F.; COELHO, R. D. Simulating wind effect on microsprinkler water distribution. Scientia Agricola, Piracicaba, v. 60, n. 2, p. 205-209, 2003.

DANTAS NETO, J.; MEDEIROS, M. G. A.; AZEVEDO, C. A. V.; AZEVEDO, H. M.
Performance hidráulica e perfil de distribuição de água do microaspersor NAAN 7110, sob diferentes condições de vento. Revista Brasileira de Engenharia Agrícola e Ambiental, Campina Grande, v.1, p.57-61, 1997.

FRIZZONE, J. A.; FREITAS, P. S. L.; REZENDE, R.; FARIA, M. A. Microirrigação: gotejamento e microaspersão.Maringá: Eduem, 2012. 356 p.

GAT, Y. L.; MOLLE, B. Model of water application under pivot sprinkler. I Theoretical grounds. Journal of Irrigation and Drainage Engineering, New York, v. 126, n. 6, p. 343-347, 2000.

INTERNATIONAL ORGANIZATION FOR STANDARDIZATION. ISO 8026. Agricultural irrigation equipment Sprayers: General requirements and test methods. Switzerland, 1995. 11p.

KELLER, J.; BLIESNER, R. D. Sprinkle and trickle irrigation. New York: Van Nostrand Reinnhold, 1990. 652 p.

MACEDO. A. B. M.; GOMES FILHO, R. R.; LIMA, S. C. R. V.; VALNIR JÚNIOR, M.; CAVALCANTE JÚNIOR, J. A. H. ARAÚJO, H. F. Desempenho Hidráulico de um sistema de irrigação por microaspersão utilizando dois tipos de emissores. Revista Brasileira de Agricultura Irrigada, Fortaleza, v. 4, n. 2, p. 82-86, 2010.

MONTERO, J.; TARJUELO, J. M.; CARRIÓN, P. SIRIAS: a simulation model for sprinkler irrigation, part II: calibration and validation or the model. Irrigation Science, New York, v. 126, n. 20, p. 85-98, 2001.

MELLO, C. R.; LIMA, J. M.; SILVA, A. M.; MELLO, J. M.; OLIVEIRA, M. S. Krigagem e inverso do quadrado da distância para interpolação dos parâmetros da equação de chuvas intensas. Revista Brasileira de Ciência do Solo, Viçosa, v. 27, p. 925-933, 2003. 
NASCIMENTO, T.; SOARES. J. M.; AZEVEDO. C. A. V. Uniformidade de distribuição de água na superfície e no perfil do solo irrigado com microaspersor RAINBIRD QN-14. Revista Brasileira de Engenharia Agrícola e Ambiental, v.13, n. 3, p.304-308, 1999.

PRADO, G. Water distribution from médiumsize sprinkler in solid set sprinkler systems. Revista Brasileira de Engenharia Agrícola e Ambiental, Campina Grande, v. 20, n. 3, p. 195-201, 2016.

PRADO, G.; COLOMBO, A. Caracterização técnica do aspersor PLONA-RL300. Irriga, Botucatu, v. 10, n. 1, p. 53-63, 2005.

PRADO, G.; COLOMBO, A. Interpolação de perfis radiais de distribuição de água de aspersores. Revista Brasileira de Engenharia Agrícola e Ambiental, Campina Grande v.17, n. 4, p.355-361, 2013.

PRADO, G.; COLOMBO, A.; BARRETO, A. C.; MATOS, F. A.; FERREIRA JÚNIOR, J.
J. Uniformidade de aplicação de água pelo aspersor PLONA-RL250 em sistemas estacionários de irrigação. Irriga, v.13, n. 2, p.220-234, 2008.

SANDRI, D.; MESQUITA, M.; BESSA, K. S. E.; PRADO, L. C. R. A. Influência do tempo de uso sobre as características hidráulicas do microaspersor do grupo modular. Engenharia Agrícola, Jaboticabal, v. 30, n. 6, p. 10891100, 2010.

SANTOS, M. A. L.; SANTOS, C. G.; MADALENA, J. A. S.; SANTOS, D. P. Influência da altura da e distância de barreiras físicas na distribuição de água de microaspersores. Revista Caatinga, Mossoró, v. 22, n. 1, p. 104-114, 2009.

SOLOMON, K.; BEZDEK, J. C. Characterizing sprinkler distribution patterns with a clustering algorithm. Transactions of the American Society of Agricultural Engineers, St. Joseph, v. 23, n. 4, p. 899-906, 1980. 